\title{
AN EXCEPTIONAL CASE OF QUADRUPLE RENAL ARTERIES \& TWIN RENAL VEINS WITH CROWDED HILAR ANATOMY
}

\author{
Sreekanth. T, Simmi Soni, Ibrahim Quadri, B. Venkat Nani Kumar, Mohd. Abdul Rafeeq.
}
1. Associate Professor. Department of Anatomy, Shadan Institute of Medical Sciences teaching Hospital \& Research Centre.
2. Associate Professor. Department of Anatomy, Dr. V.R. K. Medical College.
3. II Year M.B.B.S. Shadan Institute of Medical Sciences teaching Hospital \& Research Centre.
4. House Surgeon. Department of Anatomy, Shadan Institute of Medical Sciences teaching Hospital \& Research Centre.
5. Ist Year PG. Department of Anatomy, Shadan Institute of Medical Sciences teaching Hospital \& Research Centre.

\section{CORRESPONDING AUTHOR}

Sreekanth T., 237/2RT (10-3-799),

Vijayanagar Colony, Hyderabad- 500057.

E-mail: anatomysreekanth18@yahoo.com

Ph: 00919848826008.

\begin{abstract}
The renal vasculature was always a subject of variations both in the number and pattern of portal of entry into kidney and Perihilar placement of the artery, vein and pelvis. Good anatomical insight is an essential prerequisite besides the surgical expertise. The cadaveric dissection revealed a Right Kidney supplied by Quadruple renal arteries \& Drained by two renal veins. The main renal artery (MRA) was arising from antero lateral aspect and the accessory renal artery was arising from anterior aspect of aorta $1 \mathrm{~cm}$ distal to the former vessel. Only the MRA showed Fork Pattern Branching with four anterior segmental arteries and one posterior segmental artery in the pre hilar region. The first and fourth segmental arteries showed a small subsidiary branch and a large tortuous subsidiary branch respectively. The later along with the branch running downwards and lateral from lower renal artery formed a common trunk and pierced the capsule and entered into the substance of the kidney anteriorly about $2 \mathrm{~cm}$ lateral to the hilum, thus forming an aberrant artery. The upper polar artery was seen arising from the lower supra renal artery. The main renal vein (MRV), was formed by two formative tributaries of which one is larger and other being smaller. The later was seen just anterior to the third anterior segmental branch of MRA. Accessory renal vein was formed by only one tributary encircled by the fourth anterior segmental artery and posterior segmental artery. There was crowding of structures seen with altered hilar anatomy in both vertical and horizontal disposition. Such a rare combination of extra renal multiple arterio-venous variation is of worth concern to the urologists harvesting kidneys from the live donors for performing transplantation procedures. Partial nephrectomies for the hilar tumors and for Radiologists during interpretation of the angiograms.
\end{abstract}

KEY WORDS: (Main Renal Artery) (Accessory renal artery) (aberrant renal Artery) (Segmental branch) (Subsidiary branch) (Accessory renal vein) (Formatives tributaries)

INTRODUCTION: The renes / kidneys are a pair of excretory organs situated on the posterior abdominal wall occupying the epigastric, hypochondriac lumbar and umbilical regions, one on 
each side of the vertebral column, behind the peritoneum. Each kidney is bean-shaped with two poles i.e., upper and lower poles two borders, medial and lateral borders and two surfaces anterior and posterior surfaces. The medial border shows a depression, almost in the middle part called hilus or hilum. Normally the disposition of the structures at the hilum from anterior to posterior side is renal vein, renal artery, and the renal pelvis (VAP). The pelvis extends as the ureter which opens into the urinary bladder. The renal artery arises from the abdominal aorta and the renal vein drains into the inferior vena cava.

EMBRYOLOGY: The accessory renal arteries are due to the persistence of the embryonic lateral splanchnic arteries or (1) or persistence of the blood supply from lower level than normal. (2) accessory renal veins were the persistent mesonephric veins draining into the right subcardinal vein.

MATERIALS AND METHODS: An embalmed elderly male cadaver along with Routine instruments like a pair of gloves, Scalpel, Blade, Surgical forceps, Anatomical Forceps, Dissector, Metallic Scale with Calibrations were required. Kidneys are a pair of excretory organs lying over the posterior abdominal wall on either side of vertebral column retroperitoneally. The anterior abdominal wall was dissected layer by layer. The visceral organs like liver stomach intestines were all studied in Situ and dissected out. The duodenum, pancreas and spleen were all dissected away from the abdominal cavity and the peritoneum was striped to visualize the kidneys. The fat and facial from anterior surfaces of the right kidney was removed. The right renal vein opening into the inferior vena cava was identified. The renal arteries sprouting from the aorta were identified and traced towards the hilar region of the kidney.

OBSERVATION: During the routine prosection hours as a teaching programme for undergraduate medical students of $1^{\text {st }}$ year M.B.B.S at Shadan Institute of Medical Sciences Teaching Hospital \& Research Centre, the cadaveric dissection revealed right kindney supplied by quadruple renal arteries and drained by two renal veins. Two vessels were seen arising directly from aorta. The proximal vessel from the anterior lateral aspect of aorta at the level of the intervertebral disc between L1 - L2 was labeled as accessory renal artery (ARA) and it was running towards the lower portion of the hilum below the pelvis. The distance between the two arteries at their commencement was about $1 \mathrm{~cm}$. The main renal artery in the perihilar region divided into 4 anterior segmental branches and 1 posterior segmental branch, in a fork like (with common branching) pattern. Fig (1 \& 2) The first segmental branch and the fourth segmental branch showed a small subsidiary branch and a large tortuous large subsidiary branch respectively. Fig (3) The lower hilar artery (first accessory artery) had no segmental branches and was seen entering the hilum below the renal pelvis. The upper polar artery (second accessory artery) was seen originating from the lower supra renal arteries running downwards and lateral, towards the upper pole and piercing the capsule and the anterior substance of the kidney. It was interesting to see the third accessory renal artery formed by tortuous large subsidiary branch of the fourth segmental branch of the main renal artery along with the tortuous branch of lower supra renal artery. Fig (4) Both the branches together formed common trunk and pierced the capsule and the anterior substance of the kidney about $2 \mathrm{cms}$. Lateral to the hilum.

The main renal vein was seen to be formed by two formative tributaries of which one is larger and other being smaller. The small tributary was seen just anterior to the $3^{\text {rd }}$ anterior segmental branch of main renal artery and the large formative tributary was seen emerging out anterior and below the renal pelvis fig (5) accessory renal vein was formed by single large 
tributary seen in fig (5) was encircled by the fourth anterior segmental branch and posterior segmental branch of main renal artery. In fact, it was obscured by all the segmental arteries of the main renal artery (MRA).

In the dissected specimen a through observation of horizontal (anterior - posterior) disposition and vertical (form above downwards) disposition of the renal hilar structures was done. The horizontal disposition in the upper part of the hilum was arteries (anterior segmental) vein (accessory renal vein) and artery (posterior segmental) i.e., AVA in the middle part of hilum was vein (small formatives tributary), tributary of vein, lower hilar artery ie., VA. Fig (6) Only in the middle portion of the hilum the arrangement of the structures was coinciding with the normal anatomy given in the standard textbooks. As there were too many perihilar structures, the vertical (from above downwards) disposition of the structures was sequenced in three rows-proximal, intermediate and distal. In the proximal row the three segmental branches of MRA, small and large formative tributaries of MRV, lower hilar artery were seen. In the intermediate row the accessory renal vein was seen above, the fourth segmental branch of MRA was seen below. The distal row the posterior segmental branch of MRA was seen above and the renal pelvis seen below.

The pre hilar (extra parenchymal) segmental branches with subsidiary branches and lower hilar arteries along with veins and their tributaries, most of them having appreciable lumen size, present in the upper and lower parts of the hilum caused a crowding with altered hilar anatomy.

DISCUSSION: In the present case two renal arteries were supplying the kidney and their portal of entry was through the hilum the third artery entered through the upper pole and the fourth artery entered through the anterior substance of the kidney by piercing the capsule. Ronald A Bergman, reported the occurrence of accessory or supernumerary renal arteries in about $30 \%$ of cases, varying from two to four rarely five to six are also seen arising unilaterally or bilaterally. Quadruple renal arteries may exist as two hilar and two polar, three hilar and one polar, or one hilar and three polar renal arteries. Usually only one as these is large and the others are smaller and distributed to the superior or the inferior extremities of the kidney. Branches of the renal artery may perforate the substance of the kidneys instead of entering from the hilus. The accessory renal arteries are not just extra but essential, tissue - sustaining arteries and cannot be ligated without dire consequences. (4) in the present case there were two hilar, one upper polar and fourth renal artery was having its portal of entry through anterior substance of the kidney. Rajesh Khanna (performed 340 transplantations) reported a case of solitary patient with quadruple arteries who underwent successful anastomoses while performing about 29 multiple vessel renal transplants. (5) the statistics reveal the rarity in the occurrence of the present case. Mohammadali M. shoji studied arteriograms of 81 Kidneys and identified the perhilar branching pattern and morphology of main renal artery to be fork pattern (with Common Branching point) in $92.6 \%$ and a ladder pattern in $7.4 \%$ in the present case the MRA showed fork pattern type of perhilar branching into 5 segmental ( 4 anterior and 1 posterior) branches. (6) in the present case the MRA showed Fork Pattern Branching with four anterior segmental arteries and one posterior segmental artery in the pre hilar region. The first and fourth segmental arteries showed a small subsidiary branch and a large tortuous subsidiary branch respectively. 
Bilateral prehilar multiple branching of the renal arteries soon after their origin with the superior polar artery giving rise to the inferior supra renal artery was reported by RAO M. (7) in the present case the upper polar artery was seen arising from the lower supra renal artery (aberrant artery). The renal venous variations are less frequent in occurrence, about $0.8-6 \%$ mentioned in the standard textbooks. Satyapal reported to be much less, that is about $0.4 \%$, i.e., $3.3 \%$ on the right side and $2.6 \%$ on the left side. (7) Sampath Madhystha, reported a rare case of quadruple renal arteries with three renal veins and one of the artery entered the substance of the right kidney anteriorly lateral to the hilum with double ureter. (8). In the present case there were four arteries supplying the right kidney but only two veins draining it. In the present case only the fourth renal artery pierced the capsule and entered through the anterior substance of the kidney but the ureter was single. Kyle. J, Performed precise extraparenchymal renal hilar dissection on 73 fixed cadaveric kidneys and reported the vascular anatomy to be presegmental and segmental branches of the MRA which consisted of Zero, One, or Two presegmental arteries (external main renal artery branches that split into two or more segmental arteries) in $49.3 \%, 31.5 \%$ and $19.2 \%$ of the kidneys respectively. From a posterior approach, the posterior segmental artery was accessible to isolated clamping in $81.8 \%$ of the kidneys. The total accessibility rate for inferior segmental artery was $88.5 \%$ from anterior and $6.7 \%$ from a posterior surgical approach respectively. Segmental artery clamping is anatomically feasible and minimizes the number of nephrons exposed to potential ischemic injury. (9) Lynn H, Studied arterio-grams of 166 patients and reported the pre-hilar segmental branching in 17 male and 11 female patients. (10) Pick studied 430 body halves and reported multiple renal veins are present mostly on the right side upto $30 \%$ and rare on left side. In the present case the accessory renal vien was seen on the right side. (11) Digital subtract angiography is the gold standard for the detailed anatomical assessment of the renal vasculature for living donors. However multi detector - row computerized tomography (MDCT) is less invasive than digital subtract angiography and more accurate in the evaluation of the renal arterial anatomy such as the number, origin of the renal arteries, presence of the segmental branches and intrinsic renal artery disease and renal venous anatomy such as presence of accessory veins, retro aortic and circum aortic veins by using venous phase axial images in the living donors. According to Tombul ST the Pre-operative evaluation of the hilar vessel anatomy with 3-D computerized tomography is of great help to those patients undergoing Laparoscopic partial nephrectomy for hilar tumors as they come in direct contact either with the renal vein or artery or both. (12) Amanjot S. Sethi, opined that the use of a Doppler ultrasound probe during vascular dissection in Laparoscopic renal surgery is a fast, efficient and simple means of identifying the renal hilum. The meantime for the identification os all the renal vessels was 34.9 seconds, and the mean time to surgically isolate all the hilar vessels was 44.6 minutes without any technical difficulties (13).

CONCLUSION: The present case with multiple vascular structures leading to the crowding at the hilar region is a rare combination of extra renal multiple arterio-venous variation which is of worth concern to the urologists harvesting kidneys from the live donors for performing transplantation procedures, partial nephrectomies for the hilar tumors and for Radiologists during interpretation of the angiograms. A vast knowledge of not only frequent but also rarely occurring anatomical variations is just the beginning of perfection for an expert medical personnel including Nephrologists performing Renal transplants and Radiologist involved in Pre-operative Assessment of the patient \& as it enables them to be confident, rather than be surprised when an unusual situation arises. In the era of machine mission with multiple 
noninvasive latest modalities like Doppler Ultra sound which is an cost effective technique such rare and challenging variation can be discovered during the pre operative assessment itself enabling the surgeon for a better and safer surgery.

ACKNOWLEDGEMENTS: Thanks to The Managing Director, Dr. Sarib Rasool Khan, (SIMS) a Towering personality, for his strong Motivation towards the research work.

Thanks to Mr. M.A. Rahman Shareef, Retd., Deputy Director of Medical \& Health Sciences, Hyderabad, Andhra Pradesh. For his Inspiration and encouragement in the academic front.

Thanks to J. George William, Librarian (SIMS) \& Family for all the patience and perseverance exhibited during the typing of the manuscript.

Thanks to Mohd Ismail 3RD year M.B.BS (SIMS) for his cooperation.

Thanks to all the M.B.B.S students of 2010 batch (SIMS) for their support.

\section{REVIEW OF LITERATURE:}

1. Grays Anatomy - Churchill Livingstone 38th Edn., Peter L. Williams, Lawrence H. Bannister, Martin M beery Patricia Collins; mary Dyson. Julian E Dussek. Mark W. J. Ferguson. Pg., 1815-1836.

2. T.W Saddler, Wolters Kluwer. Lang Mans Medical Embryology $11^{\text {th }}$ Edn., Lippincott; Williams \& Wilkins. Pg 235-237

3. Ozkhan U, Oguzkurt L, Tercan F, Kizilklic O, Kocz, Koca N Renal artery orgins and variations: angiographic evaluation of 855 consecutive Patienns. Diagn Inerv radiol; 2008 12: 183-188

4. Ronald A. Bergman, PhD, Adel K. Afifi, MD, MS Ryosuke Miyauchi, MD. Anatomy. Illustrated Encyclopedia of Human Anatomic Variation: Opus II: Cardiovascular system: Arteries: Abdomen. Atlases A digital library of anatomy information

5. Rajesh Khanna, R Sood, P Madhusoodanan, T Sinha, As Sandhu, Sk Gupta, AA Pradhan, A kumar, GS Srivastava. Department of Urology, Army Hospital (Research and Referral), Delhi Cantt, India. Multiple vessel renal transplant - army hospital (research and referral) experience Indian Journal of Urology, year 2004, Volume:20 Issue: 2 Page 130 133.

6. Mohammadali M. Shoja, R. Shane Tubbs, Abolhassan Shakeri, Marios Loukas, Mohammad R. Ardalan, Hamid T. Khosroshahi and W. Jerry Oakes. Surgical and Radiologic Anatomy, Volume: 30, Number 5, July, 2008, Pg: 375-382

7. Satyapal, Rao M, Bhat SM Venkataramana V, Deepthinath R, Bolla SR. Bilateral Prehilar multiple branching of renal arteries: A case report and literature review. Kathmandu University Medical Journal (2006), Volume 4, No.3 Issue 15, 345 - 348

8. Sampath Madhyastha, R Suresh, Ramesh Rao. Department of Anatomy, Kasturba Medical College, Manipal, India. Multiple variations of renal vessels and ureter. Indian Journal of Urology, Year 2001, Volume: 17 Issue: 2 page 164-165

9. Kyle J, Weld, Sam B. Bhayani, Jay Belani, Carroline D. Ames, Greg Hruby and Jaime Landman. Extra renal Vascular Anatomy of Kidney: Assessment of Variations and their Relevance to Partial Nephrectomy From the Division of Urology, Washington University School of Medicine, St. Louis Missouri. Urology 66: 985-989, 2005. 
10. Lynn H. Harrison, Jr., M.D., M. Wayne Flye M., H.F Seigler, M.D. Incidence of Anatomical Variants in Renal Vasculature in the Presence of Normal Renal Funcition. From the Department of Surgery, Duke University Medical Centre, Durham, North Carolina. Ann. Surgery July 1978; Pg: 83-89

11. Pick, J.W and Anson, B.J: The Renal Vascular Pedicle: An Anatomical Study of 430 body Halves. J. Urology 44: 41, 1940.

12. Tombul ST; Aki FT; Gunay M; Inci K; Hazirolan T; Karaaltincaba M; Erkan I; Bakkaloglu A; Yasavul V; Preoperative evaluation of hilar vessel anatomy with 3-D computerized tomography in living kidney donors. Tombul ST- Transplant Proc - 01-Jan-2008; 40 (1): 47-9

13. Amanjot S. Sethi, M.D., Stanton M. Regan, M.D, and Chandru P. Sundaram, M.D The use of A Doppler Ultrasound Probe During Vascular Dissection in Laroscopic Renal Surgery. Journal of Endourology Volume; 23 Number 9; September 2009.

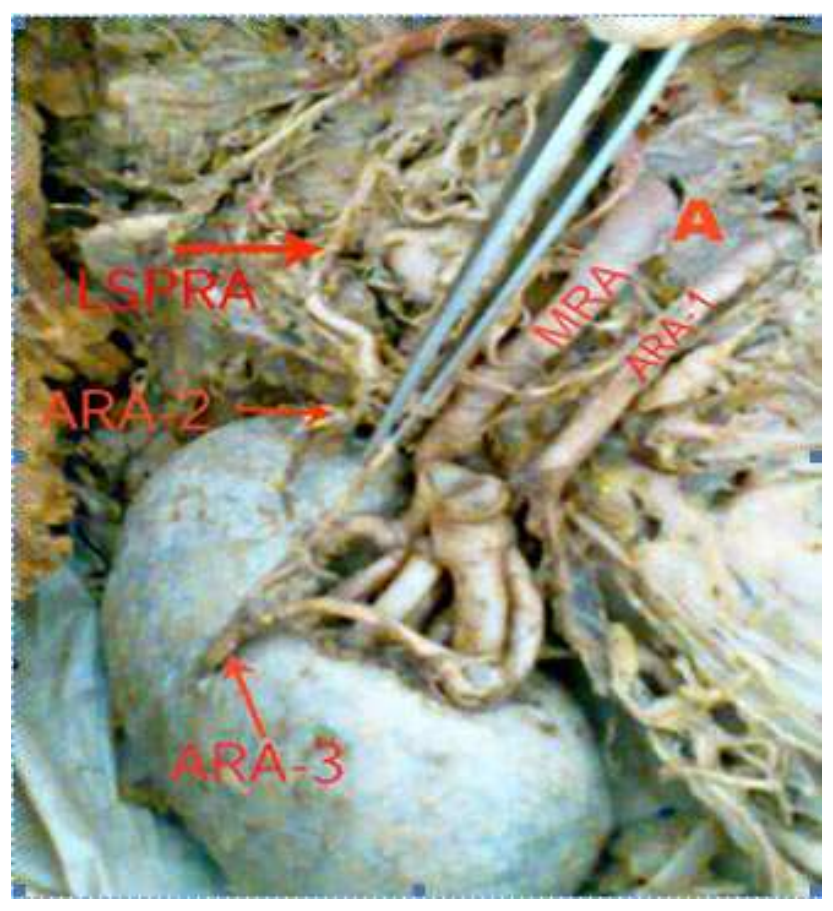

Fig (1) QUADRUPLE RENAL ARTERIES SUPPLYING THE RIGHT KIDNEY

A-AORTA, LSPRA-LOWER SUPRA RENAL ARTERY,MRA- Main Renal artery ARA -1 Accessory Renal Artery - 1 UPA / ARA2 Upper Polar artery / Accessory Renal Artery - 2 ARA -3 Accessory Renal Artery - 3 (TOTAL - 4) 


\section{CASE REPORT}

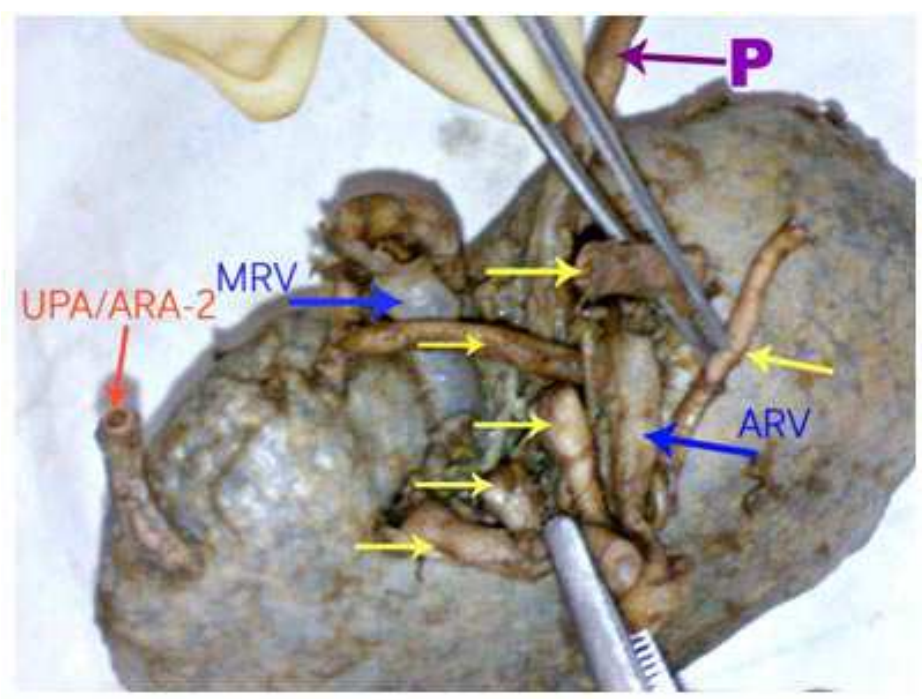

Fig (2)

MRV-MAIN RENAL VEIN, ARV- accessory renal vein P- renal pelvis(ureter) Numerous segmental branches(represented by yellow arrows), Tributaries of main renal vein and accessory renal vein causing the crowding of the hilar structures

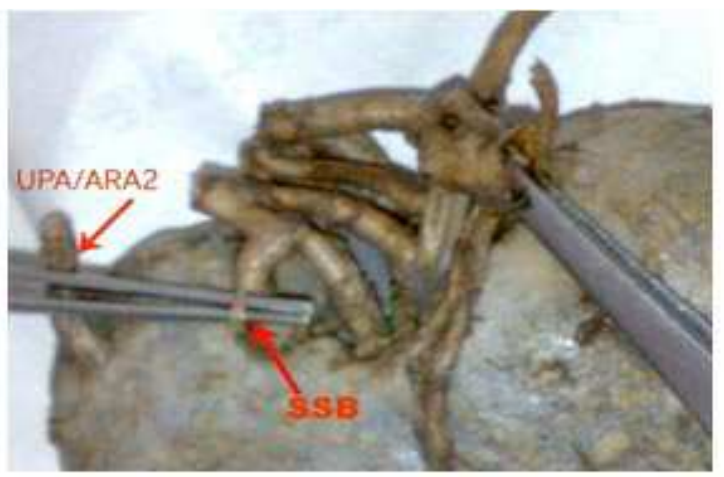

Fig (3)SSB Subsidary Branch arising from the segmental branch UPA / ARA - 2 Upper polar artery / accessory renal artery -2

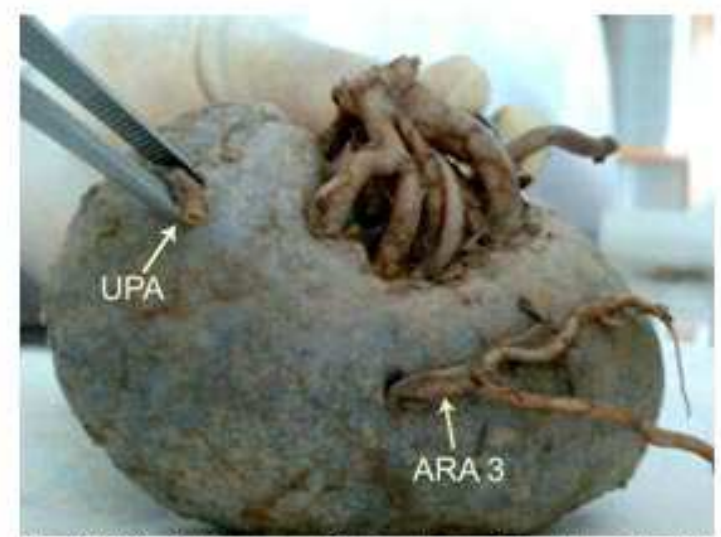

Fig (4)ARA - 3 Accessory Renal Artery - 3 entering the kidney anteriorly by piercing its capsule, UPA-upper polar artery. UPA / ARA - 2 Upper polar artery / accessory renal artery -2 


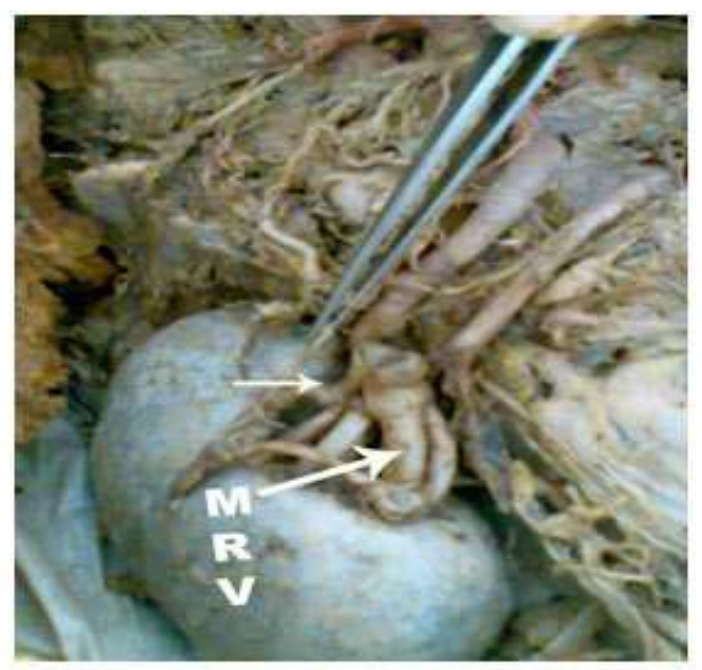

Fig (5)Main Renal Vein $\{$ which has been dissected off $\}$ Large Arrow indicating Large Tributary and small arrow indicating Smaller Tributary

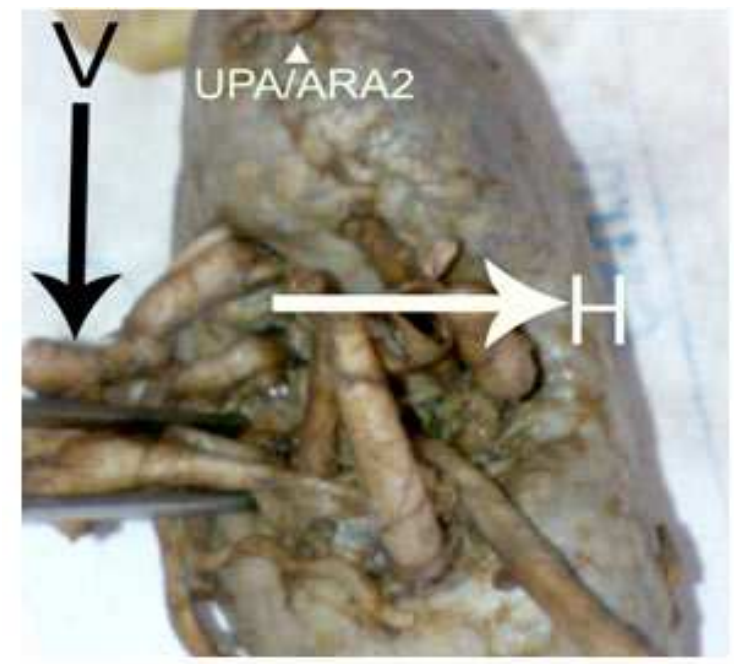

Fig (6) H- Horizontal disposition of the hilar structure V-Vertical disposition of the hilar structures 